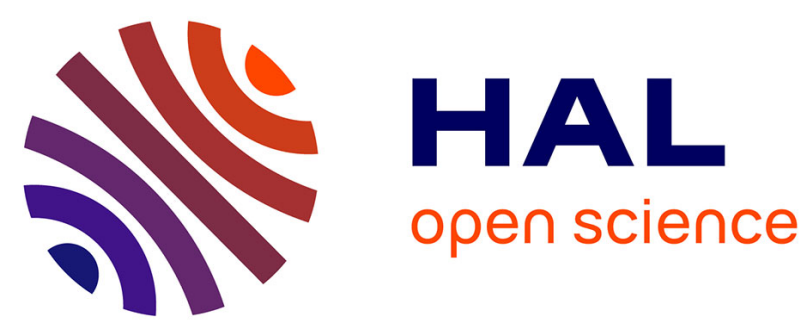

\title{
Impacts of the transmitter signal key parameters on the Compressed Sensing spectrum reconstruction for IoT Cognitive Radio applications
}

\author{
Lap Nguyen, Anthony Fiche, Roland Gautier, Emanuel Radoi
}

\section{To cite this version:}

Lap Nguyen, Anthony Fiche, Roland Gautier, Emanuel Radoi. Impacts of the transmitter signal key parameters on the Compressed Sensing spectrum reconstruction for IoT Cognitive Radio applications. Asian-Pacific Conference on Communications, Nov 2019, Ho-Chi-Minh City, Vietnam. hal-02374263

\section{HAL Id: hal-02374263 \\ https://hal.science/hal-02374263}

Submitted on 21 Nov 2019

HAL is a multi-disciplinary open access archive for the deposit and dissemination of scientific research documents, whether they are published or not. The documents may come from teaching and research institutions in France or abroad, or from public or private research centers.
L'archive ouverte pluridisciplinaire HAL, est destinée au dépôt et à la diffusion de documents scientifiques de niveau recherche, publiés ou non, émanant des établissements d'enseignement et de recherche français ou étrangers, des laboratoires publics ou privés. 


\title{
Impacts of the transmitter signal key parameters on the Compressed Sensing spectrum reconstruction for IoT Cognitive Radio applications
}

\author{
Lap Luat Nguyen, Anthony Fiche, Roland Gautier and Emanuel Radoi \\ Univ Brest, Lab-STICC, CNRS, UMR 6285, F-29200, Brest, France \\ Email: \{firstname.name\}@univ-brest.fr
}

\begin{abstract}
This paper deals with the influence of a wideband signal key parameters on its spectrum reconstruction, for Internetof-Things (IoT) applications. The impact of the frequency resolution, the bandwidths of the sensed transmitters, and the frequency spacing between their subbands are thoroughly analyzed. The spectrum detection of LoRaWAN EU868, one of the IoT standards, is simulated and examined. In the framework of cognitive networks or spectrum sensing, an input wideband signal, whose bandwidth is exceptionally large (resulting in an extremely high Nyquist rate), can be sampled at a much lower rate than the Nyquist limit. Under the hypothesis of Compressed Sensing, this paper deploys a sub-Nyquist sampling scheme, called Modulated Wideband Converter (MWC). The spectrum reconstruction is carried out from the sub-Nyquist samples, and then evaluated based on the correct reconstruction and false alarm rates.
\end{abstract}

Index Terms-Modulated Wideband Converter, spectrum sensing, sub-Nyquist sampling, frequency resolution, frequency bandwidth, subbands spacing.

\section{INTRODUCTION}

The exponential growth of wireless hardware leads to the shortage of spectrum resources to serve for application demands. Especially, in the Internet-of-Things (IoT) applications such as Smart City, a lot of wireless devices need to share a specific spectrum. It can be saturated for high access demand, while other spectrum bands are idle or vacant, but cannot be used. In this context, cognitive networks and spectrum sensing for IoT applications are proposed to re-use the licensed spectrum which is not assigned to an owner or is not being used by its owner. Hence, the applications of cognitive networks or spectrum sensing need to work on a large bandwidth to seek for the active channels or active subbands. According to the Nyquist-Shannon theorem [1] [2], the sensed signal needs to be sampled at a rate at least twice the input bandwidth to ensure perfect reconstruction. A commercial analog-to-digital converter (ADC) sampling rate cannot adapt with a very large bandwidth of a spectrum sensing application, which is usually up to several tens GHz. Consequently, Compressed Sensing [3] [4] is valuable in reducing sampling rate due to the reconstruction from sub-Nyquist samples under the sparsity assumption. Sub-Nyquist sampling techniques, in literature, have been proposed such as multicoset sampling [5], random demodulator [6] and Modulated Wideband Converter (MWC) [7].
The MWC is considered as the most practical sub-Nyquist sampling system, since it has been implemented into analog front-end hardware [8]. The MWC is a multi-physical channels system, each of them including a mixer, a lowpass filter and a conventional ADC. This system is a blind detector, having no knowledge of the input signal frequency support, which is only supposed to be sparse. To adapt with the post-processing or format of the post-hardware/software, however, the specific number of sub-Nyquist samples and specific features of input signal, which can ensure correct reconstruction, need to be studied.

The significant reduction of the MWC sampling rate results in a meaningful lowering of its cost, power consumption and complexity hardware. The IoT is currently considered as a Low Power Wide Area (LPWA) network since it usually consists in deploying low power wireless devices over a wide area network [9]. In this paper, the MWC is dedicated to detect the spectral occupancy of some common IoT standards which are being implemented on cellular network such as SIGFOX [10], LoRaWAN [11] (operate on sub-GHz) and NB-IoT [12] (coexists with LTE network). To demonstrate this capability, the simulation scenarios are set to adapt with the parameters of these standards. The frequency bands of these standards are sub-GHz. For the NB-IoT standard, the transmitter bandwidth is $180 \mathrm{kHz}$ and the spacing between transmitters is $15 \mathrm{kHz}$. The bandwidth of LoRaWAN standard is $125-500 \mathrm{kHz}$ while it is $200 \mathrm{kHz}$ in SIGFOX. We aim detecting the spectra in LoRaWAN EU868 standard which is deployed in Europe and Vietnam as well. Therefore, all the scenarios are simulated with $1 \mathrm{GHz}$ sensed frequency band and the key parameters such as transmitter bandwidth, frequency resolution and transmitter subbands frequency spacing bandwidth are changed to evaluate the spectrum reconstructions.

The method to estimate the frequency supports of wideband signal based on the minimal of each single band and the smallest spacing between bands has been proposed in [13]. In this paper, the problem is addressed in a different way. The MWC scheme is implemented in simulation to detect the wideband signal and then from the MWC output sub-Nyquist samples, the reconstruction will be made. We consider two scenarios of simulation. Firstly, the minimum bandwidth of one transmitter that the system can perfectly detect is examined with respect 
to both Nyquist and sub-Nyquist number of samples. Then, this minimum bandwidth is fixed to search for a maximum number of active subbands in that bandwidth, i.e. the number transmitters that can be detected belonging to this width. The number of transmitters, however, must respect the sparsity condition of Compressed Sensing and MWC system [14]. In the second scenario, the frequency resolution is changed to observe the impact on the spectrum reconstruction in terms of Signalto-Noise Ratio (SNR) levels. Moreover, the frequency spacing between two subbands increases gradually. This approach is intended to search for the minimum frequency spacing and the frequency resolution that allow the system to detect two adjacent subbands correctly in the presence of noise. The results are provided in terms of correct reconstruction and false alarm rates.

This paper is organized as follows. Section II-A is a brief introduction about the MWC and post-processing steps. In Section III, the minimum bandwidth of one transmitter that can be detected by the MWC is found out. Section IV presents the number of transmitters and cumulative bandwidth in one and several active subbands which can be correctly detected. Section $\mathrm{V}$ investigates the minimum frequency spacing between two adjacent subbands and examines the spectrum reconstruction of LoRaWAN EU868 standard. The conclusion is given in Section VI.

\section{The Modulated Wideband CONVERTER}

\section{A. Operating principles and system description}

The block diagram of the MWC is represented in Fig. 1. The signal $x(t)$ is considered as a multi-band signal, having an unknown, sparse spectrum. The MWC consists of $M$ physical channels. The input signal is multiplied with pseudo-random mixing sequence $p_{i}(t)$. The idea of mixing function $p_{i}(t)$ is to shift all useful subbands into baseband. Then, the lowpass filter, with the cutoff frequency $F_{c}=F_{s} / 2$, stores only the baseband input signal content. The signal in this stage is confined in the interval $\left[-F_{s} / 2 ; F_{s} / 2\right]$ width. Let us assume, for convenience, that the ADC converts the filtered signal into the digital domain using a sampling rate of $F_{s}$.

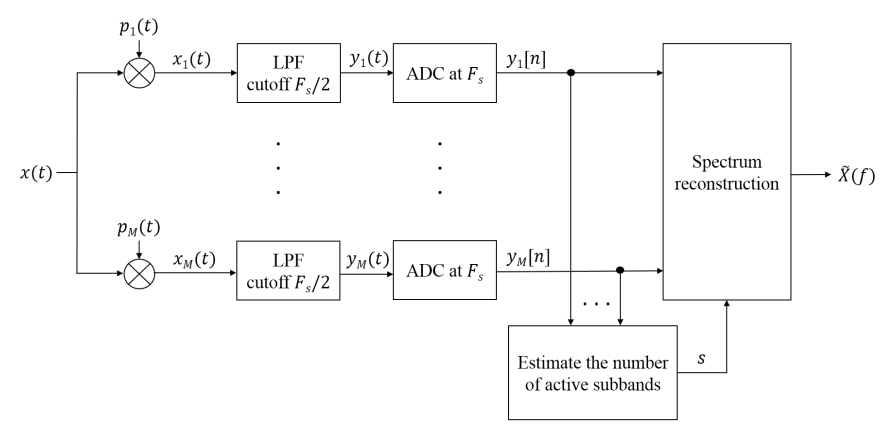

Fig. 1: The MWC scheme and processing stages.

The mixing function $p_{i}(t)$ is chosen as a periodic pseudorandom waveform, with the period $T_{p}$. The signal $x_{i}(t)$ after the mixer is

$$
x_{i}(t)=p_{i}(t) x(t),
$$

and the signal $y_{i}(t)$ after the lowpass filter reads as

$$
y_{i}(t)=h(t) * x_{i}(t),
$$

with $h(t)$ the transfer function of the lowpass filter. Finally, the digital signal is given by

$$
y[n]=y_{i}\left(n T_{s}\right),
$$

with $T_{s}$ the sampling period. In the Nyquist bandwidth, let us denote that $L$ the ratio between $F_{N y q}$ and $F_{p}$, with $L=$ $F_{N y q} / F_{p}$. It means that in the Nyquist bandwidth, there are $L$ subbands with $F_{p}$ bandwidth. The main goal of the MWC is to detect the active subbands $l$ where the transmitters are located in, with $L=2 L_{0}+1$ and $-L_{0} \leq l \leq L_{0}$.

In principle, at each physical channel of the MWC, there is one transmitter falling in the baseband $l^{\text {th }}$ subband. Hence, the higher the number of MWC physical channels $M$, the larger the number of transmitters able to be detected by the MWC. In practice, however, the MWC becomes significantly more expensive as $M$ is getting larger. Consequently, a collapsing factor $q$ is proposed to collapse the number of physical channels $M$. Instead of sampling $M$ physical channels at $F_{s}$, the signal will be considered as if $q \times M$ channels are sampled at $F_{p}$, with $q=F_{s} / F_{p}$ [14]. Theoretically, the number of physical channels of the MWC is extended to $q \times M$ channels. Then, the maximum number of transmitters that can be detected by the MWC increases to $q \times M$ transmitters. In the simulation, assuming that all the components of the MWC are ideal, the parameters of the MWC are chosen as $M=4, L=192, q=7$ and $F_{N y q}=2 \mathrm{GHz}$, so that to have a configuration compatible with LoRaWAN standard.

\section{B. Number of active subbands estimation}

In this subsection, we introduce the estimation method for the number of active channels. This task is considered as a preprocessing step in Fig. 1, before the spectrum reconstruction. In the output of the MWC, the vector $y_{i}[n](1 \leq i \leq M)$ has $N$ sub-Nyquist samples. Then, this output is considered as a $M \times N$ matrix $\mathbf{y}$, or $q M \times N$ in the case of using collapsing factor $q$.

The method to estimate the number of active subbands is based on the singular values of matrix $\mathbf{y} \in \mathbb{C}^{M \times N}$, or equivalently the associated autocorrelation matrix eigenvalues. Indeed, their variation is directly related to the number of active subbands $s$. Moreover, there are exactly $s$ non-zero eigenvalues in the noiseless case. In the case of additive white Gaussian noise, all the $M-s$ or $q M-s$ (using collapsing factor $q$ ) smallest values are equal to the noise variance. In the case of very strong noise, it is difficult to determine exactly the number of active subbands.

In the simulations, the number of active subbands $s$ is estimated using the method proposed in [15]. 


\section{Spectrum reconstruction}

A sensing matrix is formed from the periodic pseudorandom mixing sequences $p_{i}(t)$, such as $(\mathbf{P})_{i l}=p_{i l}$ [7], with $p_{i l}=F_{p} \int_{-T_{p} / 2}^{T_{p} / 2} p_{i}(t) e^{-j 2 \pi l F_{p} t} d t$ the Fourier coefficient derived from $p_{i}(t)$. Let us denote by $z_{l}[n], l=1 . . L$, the Fourier transform of the multi-subband input signal in the $l^{\text {th }}$ subband. Consequently, the system equation [8] [14] is $\mathbf{y}=\mathbf{P z}$, with $\mathbf{P} \in \mathbb{C}^{q M \times L}$.

This system equation complies with the Compressed Sensing main constraint $s \leq M q-1$, with $s$ is the number of active subbands or the non-zero elements in $\mathbf{z}$. The size of output $\mathbf{y}$ $(q \times M)$ is much smaller than the size of input $\mathbf{z}$ ( $L$ elements). The reconstruction principle is to search $\mathbf{z}$ from $\mathbf{y}$. The methods to solve such problem are called greedy algorithms or iterative methods [16] [17]. In the simulation, the greedy algorithm Orthogonal Matching Pursuit (OMP) [18] is used. To reach the solution vector, the process is divided into many iterations. In the first iteration, the unknown vector $\mathbf{z}$ is assumed to have only null elements. Then, the column $\mathbf{P}_{j}$ of matrix $\mathbf{P}$, which is most correlated to the measurement vector $\mathbf{y}$, is searched. At each iteration, the updated solution vector is found by adding nonzero components to the previously obtained vector $z$, so that to minimize the norm of the residual vector $\mathbf{r}=\mathbf{z}-\mathbf{P}_{j} \mathbf{y}$, which measures the reconstruction error. The iterations are stopped when $s$ non-zero elements are added.

\section{Minimum BANDWIDTH OF ONE TRANSMITTER}

For all simulation scenarios, the parameters of the MWC are chosen as $M=4, L=192, q=7$ and $F_{N y q}=2 \mathrm{GHz}$. Then, the other parameters can be derived: the repetition frequency of mixing function $F_{p}=F_{N y q} / L=10.4 \mathrm{MHz}$, the sampling frequency $F_{s}=q \times F_{p}=72.8 \mathrm{MHz}$, the cutoff of lowpass filter $F_{c}=F_{s} / 2=36.4 \mathrm{MHz}$.

In the first scenario, the number of samples of the wideband input signal sampled at the Nyquist rate is chosen as $N_{e}=2^{n} \times L$, in which $2^{n}$ is for the efficiency of Fast Fourier transform. Firstly, $n=11$ is chosen, resulting in $N_{e}=393216$ samples. Since $F_{s}=\frac{q \times F_{N y q}}{L}$, then, the subNyquist number of samples at MWC output, which is sampled at $F_{s}$, is $N=\frac{q \times N e}{L}=14336$ samples for each physical channel of the MWC. Overall, the number of samples at MWC output is $N_{\text {total }}=N \times M=57344$ samples. It is easy to observe that the sampling rate is reduced by a factor $r=\frac{F_{N y q}}{F_{s} \times M}=\frac{L}{q \times M}$, and in this case, the sampling rate is reduced 6.9 times at the output of the MWC. By estimating the percentage of correct detection subbands, the correct reconstruction rate is calculated by $P_{c}=\frac{\%\left(B_{r} \bigcap B_{d}\right)}{\% B_{r}}$, with $B_{r}$ the real subbands from input signal and $B_{d}$ the detected subbands. The false alarm rate is calculated by $P_{f}=\frac{\%\left(\left(B_{d} \backslash B_{r}\right) \cap \bar{B}_{r}\right)}{1-\% B_{r}}$.

The frequency resolution in this simulation is $R_{f}=\frac{F_{N y q}}{N_{e}}=$ $5 \mathrm{kHz}$. One transmitter is randomly generated in $[0 ; 1] \mathrm{GHz}$ and its bandwidth is changed. Fig. 2 shows the correct reconstruction and false alarm rates at each SNR level as a function of the transmitter bandwidth $B$ from $5 \mathrm{kHz}$ to $500 \mathrm{kHz}$. It can be observed in Fig. 2 that for a small bandwidth $5 \mathrm{kHz}$, which is equal to the frequency resolution, the correct reconstruction rate is low. This rate increases when the bandwidth increases. For high SNRs (25 and $30 \mathrm{~dB}$ ), the correct reconstruction rate can approach $100 \%$ with input bandwidth more than $250 \mathrm{kHz}$ while there is no false alarm for $30 \mathrm{~dB}$ and low false alarm rate for $25 \mathrm{~dB}$ (less than 2\%). At low $\mathrm{SNR}=15 \mathrm{~dB}$, the minimum bandwidth raises up to $380 \mathrm{kHz}$ to achieve $98 \%$ of reconstruction, however, the false alarm rate is high for this bandwidth as well.
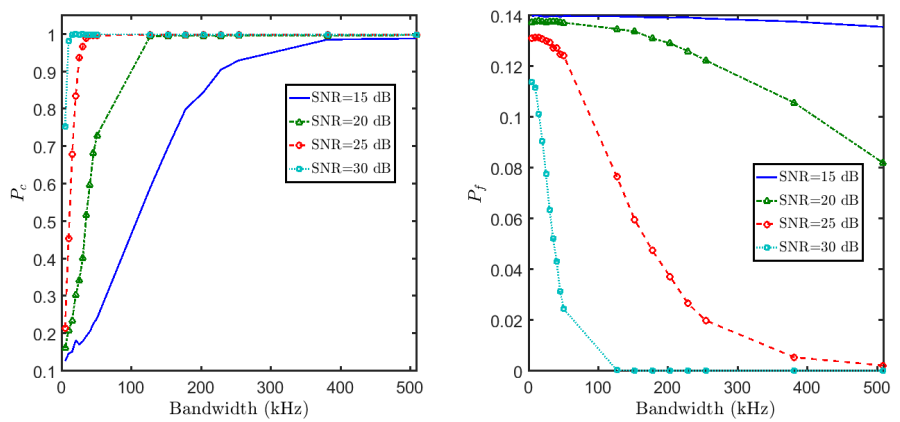

Fig. 2: Correct reconstruction and false alarm rates in function of bandwidths at $5 \mathrm{kHz}$ frequency resolution.

It should be noted that in all the simulations the SNR is evaluated in each active subband of the original spectrum, so that it can be seen as the SNR in band of interest. Let us denote the SNR in the whole Nyquist bandwidth $\mathrm{SNR}_{N y q}$. The relationship between these two SNRs is

$$
\mathrm{SNR}=\mathrm{SNR}_{N y q}+10 \log \left(\frac{F_{N y q}}{2 \times N_{t} \times B}\right),
$$

with $N_{t}$ the number of transmitters, and $N_{t}=1$ for this simulation. At $\mathrm{SNR}=15 \mathrm{~dB}$ and $B=380 \mathrm{kHz}$, the $\mathrm{SNR}_{N y q}$ in the whole Nyquist bandwidth can be inferred as $-19 \mathrm{~dB}$. A very high noise level in the whole Nyquist bandwidth that makes the useful subbands to be aliased by the noise. It leads to the over estimation of the number of active subbands $s$. This explains the reason why at SNR of $15 \mathrm{~dB}$, an incorrect reconstruction and high false alarm can be obtained.

In Fig. 3, the number of Nyquist samples is reduced to $N_{e}=98304$ samples with $n=9$, which means that the frequency resolution $R_{f}=20.3 \mathrm{kHz}$ in this simulation is larger. It is easy to observe that the performance of correct reconstruction at each SNR is reduced since the number of samples is reduced. Compared to Fig. 2, the false alarm rate increases at all SNRs and all values of bandwidth. In the first approach, it can be concluded that when more samples are used, the correct reconstruction rate is improved. It means that the resolution frequency is getting better, so that the system can detect a very small bandwidth of the transmitter.

\section{NUMBER OF TRANSMITTERS}

\section{A. Number of transmitters and cumulative bandwidth in differ- ent active subbands}

The goal of this simulation scenario is to examine the number of transmitters in different active subbands, with respect 

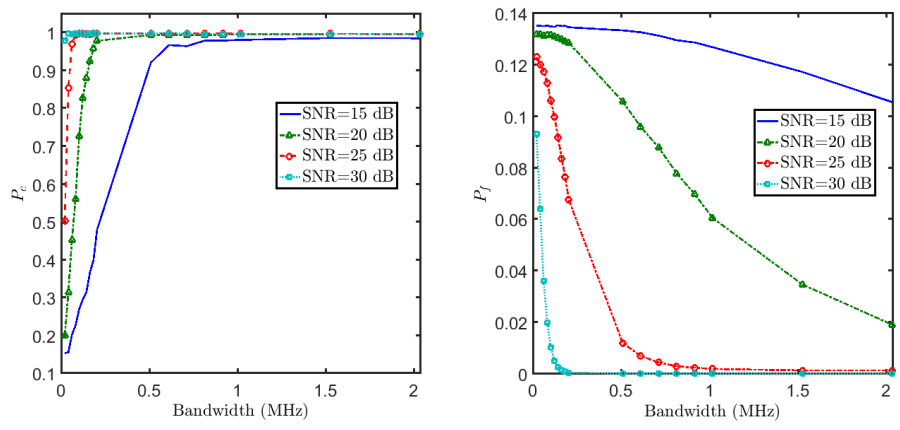

Fig. 3: Correct reconstruction and false alarm rates in function of bandwidths at $20.3 \mathrm{kHz}$ frequency resolution.

to the sparsity constraint on the number of active subbands $s \leq q M-1$ [7]. According to the MWC principle, the whole Nyquist bandwidth is divided into $L$ subbands. In this simulation, a number of transmitters $N_{t} \in[1 ; 5]$ is randomly generated in different active subbands. The number of samples is fixed at $N_{\text {total }}=57344$ samples (equivalent $N_{e}=393216$ Nyquist samples). From Fig. 2, one transmitter with $B=60$ $\mathrm{kHz}$ can be correctly detected at $\mathrm{SNR}=30 \mathrm{~dB}$. Consequently, the bandwidth of each transmitter in this simulation is chosen as $60 \mathrm{kHz}$. The MWC configurations are the same as in the previous simulations. The correct reconstruction and false alarm rates are shown in Fig. 4. To compute the correct reconstruction rate, a threshold is fixed to determine detected transmitters. In this figure, the correct reconstruction rate is reduced when increasing the number of transmitters in the case of high noise levels $(\mathrm{SNR}=15$ and $20 \mathrm{~dB})$.
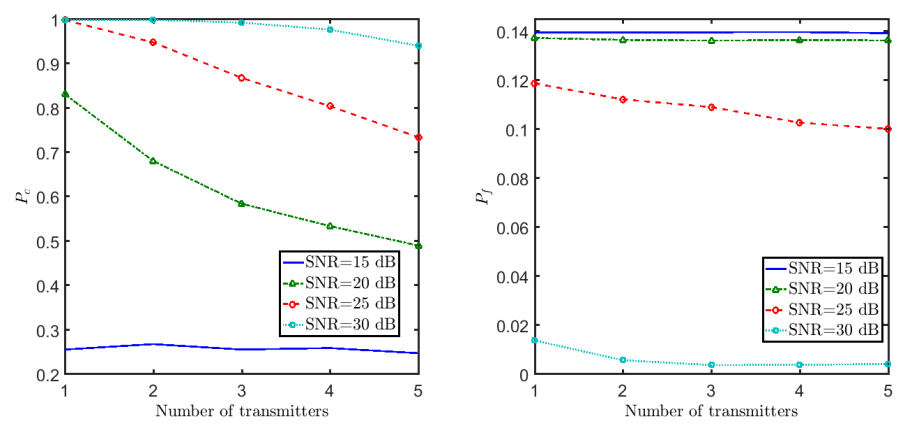

Fig. 4: Correct reconstructions and false alarm rates in function of number of transmitters in different active subbands with $B=60 \mathrm{kHz}$ and $R_{f}=5 \mathrm{kHz}$.

The transmitter bandwidths in different subbands in Fig. 4 are not accumulated. As explained in Section III, when the power of transmitter in one active subband is not significant compared to the noise, for example in case of high noise at Nyquist bandwidth $\mathrm{SNR}_{N y q}=-20 \mathrm{~dB}$ with 5 transmitters at $\mathrm{SNR}=15 \mathrm{~dB}$, it leads to a problem that the blind detection system cannot separate the transmitter from the noise.
B. Number of transmitters and cumulative bandwidth in one active subband

Fig. 5 presents the correct reconstruction and false alarm rates at each SNR level in function of number of transmitters in only one active subband. It can be seen that, the correct reconstruction rates are high even if one transmitter has narrow bandwidth $(60 \mathrm{kHz})$ except SNR $=15 \mathrm{~dB}$. When increasing the number of transmitters (up to 5 transmitters) in one subband, the correct reconstruction rate also increase. From the result of Fig. 5, the MWC system can detect 3 transmitters with 100\% of reconstruction and low false alarm (less than 5\%) at SNR of $25 \mathrm{~dB}$, equivalently $\mathrm{SNR}_{N y q}=-12 \mathrm{~dB}$.

The formula of $\mathrm{SNR}_{N y q}$ mentioned above shows that the width of detection can be accumulated by the system. If the bandwidth of a transmitter is narrow, more transmitters are needed to satisfy the requirement of signal power that the system can detect correctly. For example in Fig. 2 and Fig. 5, for a SNR of $30 \mathrm{~dB}$, the bandwidth $60 \mathrm{kHz}$ will be detected at $100 \%$ of reconstruction and no false alarm. Therefore, only one transmitter of $60 \mathrm{kHz}$ is needed in case $30 \mathrm{~dB}$ (SNR). At 25 $\mathrm{dB}$, however, $240 \mathrm{kHz}$ of bandwidth is required to be detected correctly and low false alarm (Fig. 2). Then, it needs up to 4 transmitters of $60 \mathrm{kHz}$ bandwidth (Fig. 5). It is interesting to observe that at $15 \mathrm{~dB}$, the correct reconstruction rate is high with more than 3 transmitters (Fig. 5), however, the false alarm rate is high also, it means that the system cannot separate the useful spectra and the noise. One example of spectrum reconstruction is shown in Fig. 6, where 4 transmitters with $60 \mathrm{kHz}$ bandwidth are generated consecutively at 15 and 30 $\mathrm{dB}$ in only one subband $[661.4 ; 671.8] \mathrm{MHz}$. In this case, for example in Fig. $6 \mathrm{a}(\mathrm{SNR}=15 \mathrm{~dB})$, it is difficult to discriminate the transmitters from the reconstructed signal mixed with noise. Moreover, the false alarm rate is equal to $13.9 \%$. In Fig. $6 \mathrm{~b}$ $(\mathrm{SNR}=30 \mathrm{~dB})$, all transmitters are completely detected without any false alarm. These values correspond to the false alarm rate of one transmitter with $240 \mathrm{kHz}$ bandwidth (in Fig. 2). Fig. 2 and Fig. 5 show that the bandwidth of transmitters in one active subband can be accumulated and it leads to a better reconstruction if more bandwidths are accumulated, since the proportion of signal power and noise in that band is enhanced significantly.
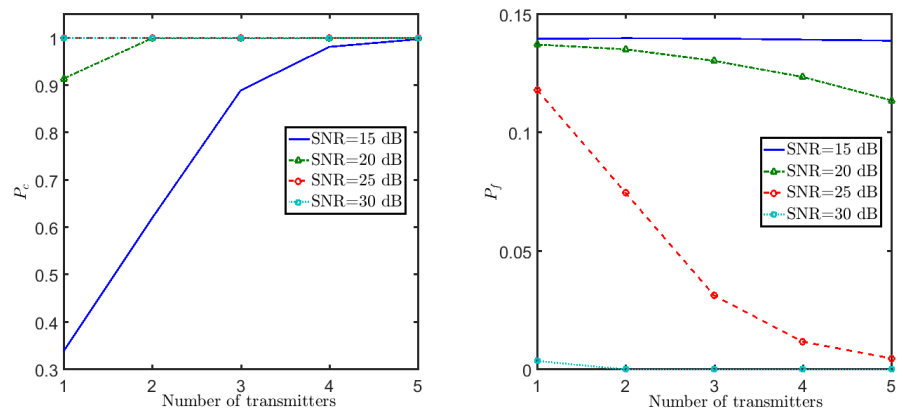

Fig. 5: Correct reconstructions and false alarm rates in function of number of transmitters in one active subband with $B=60 \mathrm{kHz}$ and $R_{f}=5 \mathrm{kHz}$. 

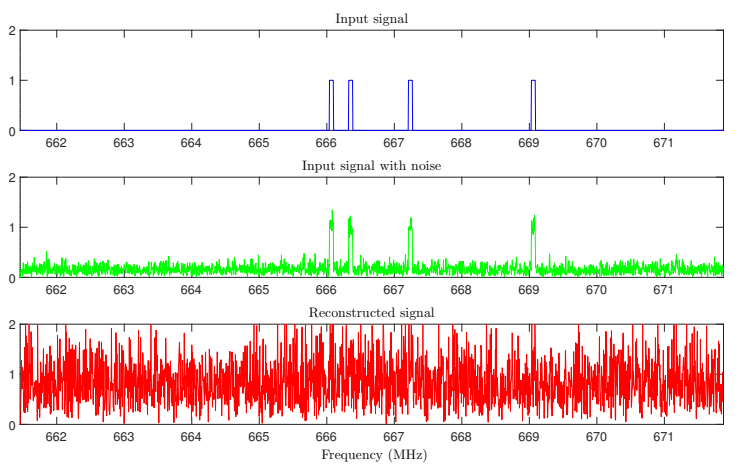

(a) $\mathrm{SNR}=15 \mathrm{~dB}$
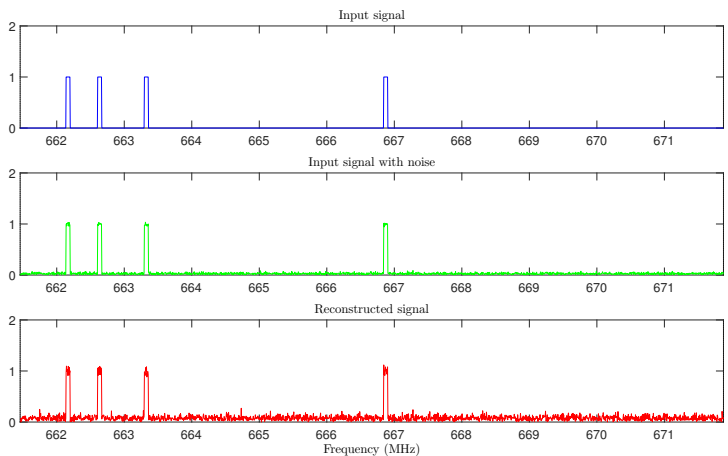

(b) $\mathrm{SNR}=30 \mathrm{~dB}$

Fig. 6: Spectrum reconstructions of 4 transmitters generated in one active subband at a SNR of 15 and $30 \mathrm{~dB}$ with $B=60 \mathrm{kHz}$ and $R_{f}=5 \mathrm{kHz}$.

\section{FREQUENCY SPACING AND LORAWAN SPECTRUM DETECTION APPLICATION}

\section{A. Minimum frequency spacing}

In this section, the frequency spacing of 4 transmitters increases gradually, from $R_{f}=5 \mathrm{kHz}$ to $8 R_{f}=40 \mathrm{kHz}$ with $B=60 \mathrm{kHz}$. The transmitters are generated consecutively in only one subband $[859.3 ; 869.7] \mathrm{MHz}$. We introduce a measurement $P_{B}$ which corresponds to the correct detection rate of the minimum and maximum frequencies $\left\{f_{t_{\text {min }}} ; f_{t_{\text {max }}}\right\}$ of all transmitter bandwidths, with $t \in\left[1 ; N_{t}\right]$.

Fig. 7 illustrates the correct detection rate of $\left\{f_{t_{\min }} ; f_{t_{\max }}\right\}$ and false alarm rate in function of frequency spacing. At high noise levels (SNR of 15 and $20 \mathrm{~dB}$ ), the reconstructions are incorrect regardless the frequency spacing between these transmitters is small or large. The high noise makes the detection of power transitions difficultly and leads to an incorrect estimation of couples $\left\{f_{t_{\min }} ; f_{t_{\max }}\right\}$. For low noise level, the reconstructions are improved. Thus, perfect reconstruction at $30 \mathrm{~dB}$ and high correct reconstruction rates at $25 \mathrm{~dB}$ can be obtained, without any false alarm. It can be inferred that the minimum frequency spacing is $5 \mathrm{kHz}$ at $\mathrm{SNR}=30$ $\mathrm{dB}$ with perfect reconstruction and at $\mathrm{SNR}=25 \mathrm{~dB}$ with high correct reconstruction rate (more than 90\%) and no false alarm. Fig. 8 confirms the simulated results with the spectrum reconstructions at a SNR of 20 and $30 \mathrm{~dB}$. The ability to discriminate two adjacent transmitters is directly related to the frequency resolution shown in Fig. 7, and that the performances of reconstruction depend only on the SNR.
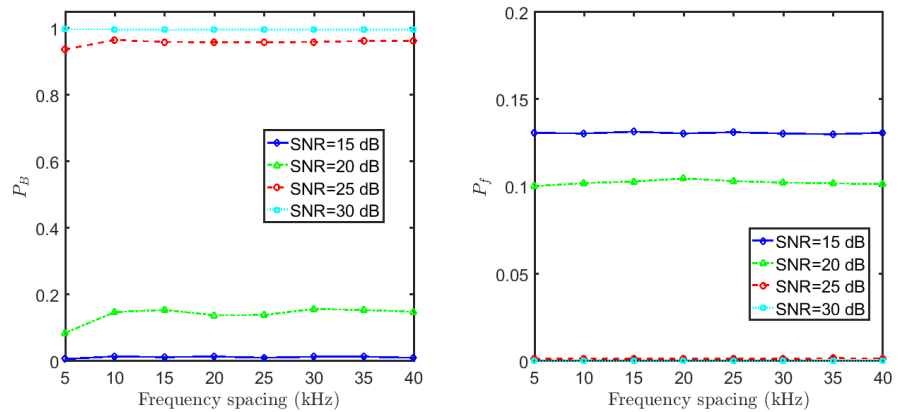

Fig. 7: Correct detection rate of all couples $\left\{f_{t_{\min }} ; f_{t_{\max }}\right\}$ and false alarm rate in function of frequency spacing with $B=60 \mathrm{kHz}$ and $R_{f}=5 \mathrm{kHz}$.

\section{B. LoRaWAN spectrum detection application}

In this section, the frequency spacing between transmitters in LoRaWAN is studied and the configuration of LoRaWAN standard is simulated to examine the spectrum detection in the IoT applications.

The LoRaWAN EU868 standard is used in the ISM band. From 867.1 to $868.5 \mathrm{MHz}$ frequency band, the LoRaWAN standard can deploy up to 8 transmitters with $125 \mathrm{kHz}$ bandwidth and $75 \mathrm{kHz}$ guard band (frequency spacing) for each transmitter [19]. The total bandwidth of all transmitters is equal to $8 \times 0.125=1 \mathrm{MHz}$. The total sub-Nyquist number of samples is $N_{\text {total }}=57434$ and Nyquist number of samples is $N_{e}=393216$. The correct reconstruction and false alarm rates of LoRaWAN EU868 standard with 8 transmitters from 867.1 to $868.5 \mathrm{MHz}$ is shown in Table I. As the simulation result from previous sections, the correct reconstruction and false alarm rates can approach $100 \%$ in the case of SNR $=30$ $\mathrm{dB}$, corresponding to $\mathrm{SNR}_{N y q}=0 \mathrm{~dB}$. Moreover, there is no correct detection in cases of very high noise $\mathrm{SNR}=15$ and $20 \mathrm{~dB}$, corresponding to $\mathrm{SNR}_{N y q}=-15$ and $-10 \mathrm{~dB}$. Fig 9 illustrates one example of LoRaWAN EU868 standard spectrum reconstruction with $30 \mathrm{~dB}$ (SNR). The spectra can be obtained with a correction of number of transmitters and frequency bands.

\begin{tabular}{|c|c|c|c|c|}
\hline SNR (dB) & 15 & 20 & 25 & 30 \\
\hline$P_{B}$ & 0.02 & 0.25 & 0.86 & 1 \\
\hline$P_{c}$ & 0.97 & 0.93 & 0.99 & 1 \\
\hline$P_{f}$ & 0.12 & 0.004 & 0 & 0 \\
\hline
\end{tabular}

TABLE I: Correct detection of $\left\{f_{t_{\min }} ; f_{t_{\max }}\right\}$, correct reconstruction and false alarm rates of 8 transmitters LoRaWAN in function of SNRs.

Overall, from the simulation results, the number of transmitters, the frequency resolution and the cumulative or noncumulative bandwidth all cause an impact on the sensed spectrum reconstruction. Nevertheless, the biggest constraint is the 

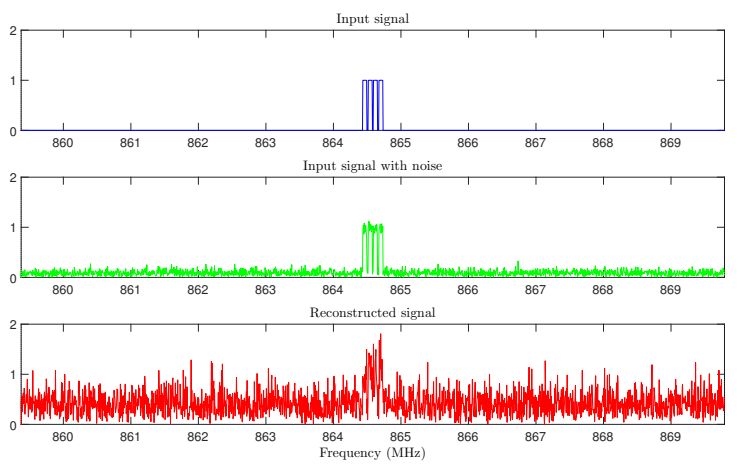

(a) $\mathrm{SNR}=20 \mathrm{~dB}$
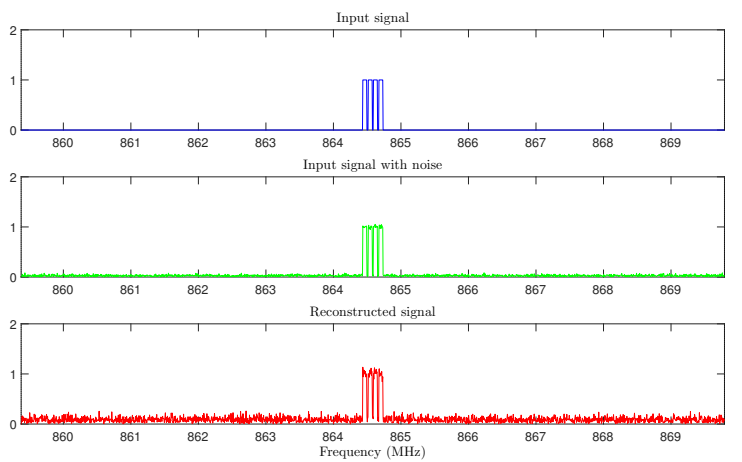

(b) $\mathrm{SNR}=30 \mathrm{~dB}$

Fig. 8: Spectrum reconstructions of 4 transmitters generated consecutively with frequency spacing $5 \mathrm{kHz}$ at a SNR of 20 and $30 \mathrm{~dB}$, $B=60 \mathrm{kHz}$ and $R_{f}=5 \mathrm{kHz}$.
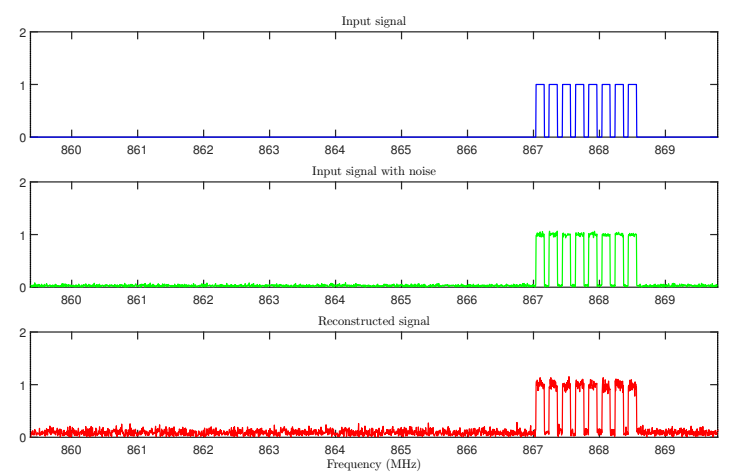

Fig. 9: Example of LoRaWAN EU868 blind spectrum reconstruction.

noise levels. The intensity of noise determines the corrections of all the spectrum reconstruction in a spectrum sensing system.

\section{CONCLUSION}

To conclude, this paper analyses the key parameters of a wideband signal and studies their impact on the reconstructions of a sub-Nyquist sampling system. With the aim of applying spectrum sensing techniques to IoT applications, this research focuses on the LoRaWAN EU868 standard and deploys the MWC sub-Nyquist sampling system because it is a low-cost, low complexity hardware and efficient in power consumption. With regard to the results, the Compressed Sensing technique can be used for IoT applications. In future work, the study on the ability of discrimination between adjacent transmitters will be improved to evaluate the limits and a particular interest must be brought to the study according to the difference of transmitted power levels. This will also allow us to parameterize a real system by being able to do a compromise between an improvement of performance and an extra computing cost (therefore an extra power consumption).

\section{ACKNOWLEDGEMENT}

This research is partly supported by the IBNM (Brest Institute of Computer Science and Mathematics) CyberIoT Chair of Excellence at the University of Brest, and by the RAPID REACC-RF project.

\section{REFERENCES}

[1] H. Nyquist, "Certain topics in telegraph transmission theory," Transactions of the American Institute of Electrical Engineers, vol. 47, no. 2, pp. 617-644, 1928.

[2] C. E. Shannon, "Communication in the presence of noise," Proceedings of the IRE, vol. 37, no. 1, pp. 10-21, 1949.

[3] E. J. Candès, J. Romberg, and T. Tao, "Robust uncertainty principles: Exact signal reconstruction from highly incomplete frequency information," IEEE Trans. Inform. Theory, vol. 52, no. 2, pp. 489-509, 2006.

[4] D. L. Donoho, "Compressed Sensing," IEEE Trans. on Inform. Theory, vol. 52, no. 4, pp. 1289-1306, 2006.

[5] M. Mishali and Y. C. Eldar, "Blind multiband signal reconstruction: Compressed sensing for analog signals," IEEE Trans. on Signal Processing, vol. 57, no. 3, pp. 993-1009, 2009.

[6] J. A. Tropp, J. N. Laska, M. F. Duarte, J. K. Romberg, and R. G. Baraniuk, "Beyond nyquist: Efficient sampling of sparse bandlimited signals," IEEE Transactions on Information Theory, vol. 56, no. 1, pp. 520-544, 2010.

[7] M. Mishali and Y. C. Eldar, "From theory to practice: Sub-nyquist sampling of sparse wideband analog signals," IEEE J. Sel. Topics in Signal Processing, vol. 4, no. 2, pp. 375-391, 2010.

[8] M. Mishali, Y. C. Eldar, O. Dounaevsky, and E. Shoshan, "Xampling: Analog to digital at sub-Nyquist rates," IET Circuits, Dev. Syst., vol. 5, pp. 8-20, Jan. 2011.

[9] U. Raza, P. Kulkarni, and M. Sooriyabandara, "Low power wide area networks: An overview," IEEE Communications Surveys \& Tutorials, vol. 19, no. 2, pp. 855-873, 2017.

[10] SIGFOX. http://www.sigfox.com, 2019.

[11] N. Sornin, M. Luis, T. Eirich, T. Kramp, and O. Hersent, "LoRaWAN Specification," LoRa Alliance, 2015.

[12] S. Grant, "3GPP Low Power Wide Area Technologies-GSMA White Paper," gsma.com, 2016.

[13] M. Mishali, A. Elron, and Y. C. Eldar, "Sub-nyquist processing with the modulated wideband converter," in 2010 IEEE Int. Conf. on Acoustics, Speech and Signal Processing, pp. 3626-3629, IEEE, 2010.

[14] M. Mishali, Y. C. Eldar, and A. J. Elron, "Xampling: Signal acquisition and processing in union of subspaces," IEEE Trans. Signal Processing, vol. 59, no. 10, pp. 4719-4734, 2011.

[15] E. Radoi and A. Quinquis, "A New Method for Estimating the Number of Harmonic Components in Noise with Application in High Resolution Radar," J. on Applied Signal Processing, pp. 1177-1188, 2004.

[16] J. A. Tropp, A. C. Gilbert, and M. J. Strauss, "Algorithms for simultaneous sparse approximation. part i: Greedy pursuit," Signal Processing, vol. 86, no. 3, pp. 572-588, 2006.

[17] J. Tropp, A. C. Gilbert, et al., "Signal recovery from partial information via orthogonal matching pursuit," IEEE Trans. Inform. Theory, vol. 53, no. 12 , pp. 4655-4666, 2007.

[18] T. T. Cai and L. Wang, "Orthogonal Matching Pursuit for sparse signal recovery with noise," IEEE Trans. on Inform. Theory, vol. 57, no. 7, pp. 4680-4688, 2011.

[19] L. A. T. C. R. P. Workgroup, "LoRaWAN 1.1 Regional Parameters," LoRa Alliance, 2018 January. 